\title{
Improved oral hygiene is associated with decreased risk of new-onset diabetes: a nationwide population-based cohort study
}

\author{
Yoonkyung Chang ${ }^{1} \cdot$ Ji Sung Lee ${ }^{2} \cdot \mathrm{Ki}^{-J u n g} \mathrm{Lee}^{3} \cdot \mathrm{Ho} \mathrm{Geol} \mathrm{Woo}^{4} \cdot$ Tae-Jin Song $^{4}$ (ID
}

Received: 14 September 2019 / Accepted: 23 January 2020 / Published online: 2 March 2020

(C) Springer-Verlag GmbH Germany, part of Springer Nature 2020

\begin{abstract}
Aims/hypothesis Inflammation plays an important role in the development of diabetes, a major global health problem. Periodontal disease is also common in the general population. Because periodontal disease and poor oral hygiene can provoke transient bacteraemia and systemic inflammation, we hypothesised that periodontal disease and oral hygiene indicators would be associated with the occurrence of new-onset diabetes.

Methods In this study we analysed data collected between 2003 and 2006 on 188,013 subjects from the National Health Insurance System-Health Screening Cohort (NHIS-HEALS) in Korea who had no missing data for demographics, past medical history, oral hygiene indicators or laboratory findings. The presence of periodontal disease was defined on the basis of a modified version of ICD-10 codes (Korean Classification of Disease, sixth edition), if claims for treatment for acute periodontitis (K052), chronic periodontitis (K053) and periodontosis (K054) were made more than two times by a dentist, or if, according to medical records, subjects received treatment by a dentist for periodontal disease with ICD-10 codes K052, K053 or K054. Oral hygiene behaviours (number of tooth brushings, a dental visit for any reason and professional dental cleaning) were collected as selfreported data of dental health check-ups. Number of missing teeth was ascertained by dentists during oral health examination. The incidence of new-onset diabetes was defined according to ICD-10 codes E10-E14. The criterial included at least one claim per year for both visiting an outpatient clinic and admission accompanying prescription records for any glucose-lowering agent, or was based on a fasting plasma glucose $\geq 7 \mathrm{mmol} / \mathrm{l}$ from NHIS-HEALS.

Results Of the included subjects, $17.5 \%$ had periodontal disease. After a median follow-up of 10.0 years, diabetes developed in 31,545 (event rate: $16.1 \%, 95 \%$ CI 15.9\%, 16.3\%) subjects. In multivariable models, after adjusting for demographics, regular exercise, alcohol consumption, smoking status, vascular risk factors, history of malignancy and laboratory findings, the presence of periodontal disease (HR 1.09, 95\% CI 1.07, 1.12, $p<0.001$ ) and number of missing teeth ( $\geq 15$ teeth) remained positively associated with occurrence of new-onset diabetes (HR 1.21, 95\% CI 1.09, 1.33, $p<0.001, p$ for trend $<0.001$ ). Frequent tooth brushing ( $\geq 3$ times/ day) was negatively associated with occurrence of new-onset diabetes (HR 0.92, 95\% CI 0.89, 0.95, $p<0.001, p$ for trend $<0.001$ ). Conclusions/interpretation Frequent tooth brushing may be an attenuating factor and the presence of periodontal disease and an increased number of missing teeth may be augmenting factors for the occurrence of new-onset diabetes. Improving oral hygiene may be associated with a decreased risk of occurrence of new-onset diabetes.
\end{abstract}

Keywords Diabetes mellitus $\cdot$ Epidemiology $\cdot$ Oral hygiene $\cdot$ Periodontal diseases $\cdot$ Periodontitis $\cdot$ Tooth loss $\cdot$ Toothbrushing

Yoonkyung Chang and Ji Sung Lee contributed equally to this study.

Electronic supplementary material The online version of this article (https://doi.org/10.1007/s00125-020-05112-9) contains peer-reviewed but unedited supplementary material, which is available to authorised users.

Tae-Jin Song

knstar@ewha.ac.kr

1 Department of Neurology, Mokdong Hospital, Ewha Womans University College of Medicine, Seoul, South Korea

2 Clinical Research Center, Asan Medical Center, Seoul, South Korea
3 Department of Neurology, Eunpyeong St Mary's Hospital, Catholic University of Korea, Seoul, South Korea

4 Department of Neurology, Seoul Hospital, Ewha Womans University College of Medicine, 260, Gonghang-daero, Gangseo-gu, 07804 Seoul, Republic of Korea 


\section{Research in context}

\section{What is already known about this subject?}

- Inflammatory reactions are an important cause of diabetes via increasing insulin resistance and endothelial dysfunction

- Because periodontal disease and poor oral hygiene can provoke transient bacteraemia and systemic inflammation, this immune process may be associated with the occurrence of new-onset diabetes

\section{What is the key question?}

- Is there an association between oral hygiene indicators and the risk of new-onset diabetes?

\section{What are the new findings?}

- $\quad$ Frequent tooth brushing was associated with a decreased risk of new-onset diabetes, while the presence of periodontal diseases and an increased number of missing teeth were positively associated with the risk of newonset diabetes

\section{How might this impact on clinical practice in the foreseeable future?}

- Improving oral hygiene, e.g. by frequent tooth brushing, may decrease the risk of new-onset diabetes

\section{Abbreviations \\ LPS Lipopolysaccharide \\ NHIS National Health Insurance System \\ NHIS-HEALS National Health Insurance \\ System-National Health Screening Cohort}

\section{Introduction}

Over the last century, diabetes has become one of the largest global health emergencies in both Eastern and Western countries $[1,2]$. Besides the well-known microvascular complications of diabetes, such as nephropathy, neuropathy and retinopathy, there is an expanding epidemic of macrovascular complications, including diseases of the carotid, cerebral, coronary and peripheral arteries [3]. Currently, several preventive strategies for newonset diabetes have been suggested that include controlling factors related to a healthy lifestyle, such as maintaining proper weight and waist circumference, increasing regular exercise or physical activity, practising healthy dietary habits and undergoing regular physical examinations for identifying risk factors [4]. However, disease-modifying drugs and preventive methods for occurrence of new-onset diabetes are still lacking.

Periodontal disease is one of the most common diseases in the general population [5], involving a set of inflammatory reactions affecting the surrounding tissues of the teeth, such as the gingiva, periodontal ligaments and alveolar bone, which may ultimately cause tooth loss and elicit systemic inflammation [6]. In addition to periodontal disease, there are also indicators, such as frequency of tooth brushing, frequency of professional dental cleaning and number of missing teeth, that are closely related to oral hygiene [7-9]. Periodontal disease and poor oral hygiene indicators are associated with cardiovascular diseases, including hypertension, stroke, myocardial infarction and long-term mortality [10, 11].

Inflammatory reactions are an important cause of diabetes via increasing insulin resistance and endothelial dysfunction [12]. In a cross-sectional population-based sample, a positive relationship between inflammation and diabetes was demonstrated [13]. Because periodontal disease is a microbially triggered chronic inflammatory disease, associated systemic inflammatory reactions from the invasion of oral bacteria and release of inflammatory mediators could affect glycaemic control [14]. It is therefore plausible that systemic and chronic inflammatory reactions derived from periodontal disease or poor oral hygiene may impact the occurrence of new-onset diabetes [14, 15]. Furthermore, paradoxically, transient bacteraemia is also caused by regular tooth brushing, even in individuals with good oral health [16]. However, studies regarding the association of periodontal disease and oral hygiene indicators with the occurrence of new-onset diabetes are lacking, particularly in a longitudinal setting.

We hypothesised that improved oral hygiene would be associated with decreased risk of new-onset diabetes and that periodontal disease and poor oral hygiene would be related to increased risk of new-onset diabetes. The main aim of this study was to characterise the relationship between periodontal disease and new-onset diabetes. The secondary aim of this study was to identify associations of other oral hygiene indicators with newonset diabetes in a nationwide population-based cohort dataset.

\section{Methods}

Data sources The National Health Insurance System (NHIS) collects information on demographics, socioeconomic status 
and type of health insurance coverage, with a medical database for diagnosis and treatment modality information. In Korea, the NHIS is the sole insurance provider, covering about $97 \%$ of the Korean population, and is controlled and supported by the Korean government. The remaining 3\% of the population is supported by the Medical Aid programme. Subscribers to the NHIS are recommended to receive standardised medical health examinations every 2 years [17]. In 2015 the NHIS constructed the NHIS-National Health Screening Cohort (NHIS-HEALS) database with the aim of making useful data available to health researchers [18]. The database contains information on 514,866 individuals through random sampling of data on 50 million individuals who attended the medical health examinations. We analysed data from this database in the present study [18]. The height, weight, laboratory tests, questionnaires on lifestyle, oral health status and oral hygiene behaviours of these individuals were all assessed by the NHIS. The standardised medical health examinations include an oral disease and oral hygiene behaviour screening programme for subscribers aged $\geq 40$ years. This screening programme consists of a self-reported questionnaire regarding information about dental symptoms, dental visits during the last year and oral hygiene behaviour and an examination by a dentist for the presence of tooth conditions, including number of missing teeth. If dental problems were present, improved oral hygiene practices were recommended [10].

Subjects As mentioned above, our study was based on data from the NHIS-HEALS database. Subjects were enrolled from 2003 to 2006 and followed up for incident diabetes until 2015. Subjects had to be free from diabetes for more than 1 year to rule out individuals with diabetes at the beginning of the study. All subjects had received routine medical examinations and information was obtained on medical histories, including age, sex, socioeconomic status, regular exercise, alcohol consumption, smoking status, BMI, blood pressure, comorbidities, laboratory tests and oral hygiene indicators. NHIS-HEALS comprised data on 514,866 individuals. In our study, among these individuals, those with missing data for variables such as oral health status $(n=296,718)$ or health examination $(n=$ $5093)$ were excluded. We also excluded individuals $(n=$ 25,042 ) who had already been diagnosed with diabetes (ICD10 codes E10-E14, http://apps.who.int/classifications/icd10/ browse/2016/en). The criteria for a prior diagnosis of diabetes was having at least one claim per year for glucose-lowering agents or at least one fasting plasma glucose $\geq 7 \mathrm{mmol} / 1$ result from the NHIS-HEALS database before the index date. Ultimately, 188,013 subjects were analysed in this study (Fig. 1). This study was approved by Ewha Womans University of College of Medicine Institutional Review Board (approval number: EUMC 2018-01-067). Informed consent was waived because retrospective anonymised data were used.

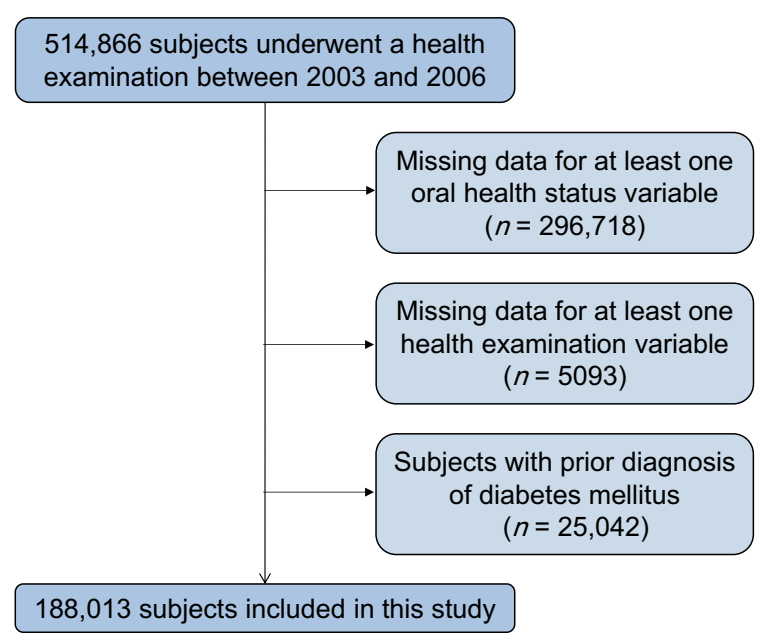

Fig. 1 Flow chart of study subjects

Definition and measurement of variables Information on smoking habits and alcohol consumption were obtained by questionnaire. BMI was defined as participant weight in kilograms divided by the square of participant height in metres. Regular physical exercise was considered to be strenuous physical activity performed for at least 20 min more than once per week [19]. Economic status was dichotomised at the bottom $10 \%$. Hypertension was defined using ICD-10 codes I10-I11 and the prescription of an antihypertensive agent with at least one claim per year. Dyslipidaemia was defined using ICD-10 code E78 and the prescription of a lipid-lowering agent, including statins, with at least one claim per year. Smoking status was categorised into none, former smoker, and current smoker. Current smoker was classified according to the WHO definition as a person who has smoked more than five packs (100 cigarettes) in a lifetime and smoked daily or occasionally for the last 28 days. Former smoker was defined as a person who had smoked more than 100 cigarettes in a lifetime and had not smoked in the last 28 days [20]. Renal disease was identified by ICD-10 codes N18.1-N18.5 and N18.9 with at least one claim per year. Malignancy was defined by ICD-10 codes C00-D48 with at least one claim per year. For BMI and blood and urine laboratory findings more than one result was available and the most recent results were used in the present study [10].

In line with previous studies [10,21,22], the presence of periodontal disease was defined as claims for treatment for the modified version of ICD-10 codes used in Korean Health insurance, Korean Classification of Disease, sixth edition (acute periodontitis [K052], chronic periodontitis [K053], and periodontosis [K054]) more than two times by a dentist or if subjects received treatment by a dentist for periodontal disease with ICD-10 codes K052, K053, and K054. Oral hygiene behaviours (frequency of tooth brushing, a dental visit for any reason, and professional dental cleaning) were collected as self-reported data of dental health check-ups 
[10]. Oral hygiene behaviour was categorised by the number of tooth brushings: $0-1$ time, 2 times and $\geq 3$ times per day. A dental visit for any reason and professional dental cleaning were dichotomised as never or at least once per year. Number of missing teeth including wisdom teeth was ascertained by dentists during oral health examinations [10, 23]. The number of missing teeth was classified as $0,1-7,8-$ 14 , and $\geq 15$ regardless of the cause (e.g. periodontal disease or other dental reason). The index date was defined as the date of the oral health status examination. If more than two dental health check-ups occurred between 2003 and 2006, the most recent results were used for the analysis.

Definition of new-onset diabetes The incidence of new-onset diabetes was defined as main or secondary diagnosis of diabetes (ICD-10 codes E10-E14). Criteria for this diagnosis included at least one claim per year for both visiting an outpatient clinic and admission accompanied by prescription records for any hypoglycaemic agents. Alternatively, at least one fasting plasma glucose $\geq 7 \mathrm{mmol} / 1$ result from NHISHEALS led to a diagnosis of new-onset diabetes [19].

Statistical analysis We performed $\chi^{2}$ tests and independent sample $t$ tests for comparison of categorical and continuous variables, respectively. To evaluate the presence of selection bias, we investigated standardised differences in demographics between included and excluded subjects. Because statistical power to detect differences is based on sample size, there is the possibility of a false positive using $\chi^{2}$ tests and independent sample $t$ tests on data from a large sample size. For this reason, standardised differences of greater than 0.1 were defined as noteworthy.

Kaplan-Meier survival curves with logrank test were used to evaluate the relationship between oral hygiene indicators and incidence of new-onset diabetes. Cox proportional hazard models were applied to determine the association of oral hygiene indicators and occurrence of diabetes; HRs and 95\% CIs were determined. Multivariable regression models were constructed with adjustment for Model 1 (age, sex, socioeconomic status, alcohol consumption, regular exercise, BMI, hypertension, dyslipidaemia, smoking status, renal disease and history of malignancy), Model 2 (Model $1+$ systolic blood pressure, fasting blood glucose, liver panel [aspartate aminotransferase, alanine aminotransferase, $\gamma$-glutamyl transferase] and presence of proteinuria), and Model 3 (Model $2+$ periodontal disease, number of tooth brushings, dental visits for any reason, professional dental cleaning and number of missing teeth). All subjects with any missing data were excluded from the statistical analysis, and uni- and multivariable analyses were performed on cases with complete data. To investigate trends for HR based on number of tooth brushings and number of missing teeth, $p$ value for trend was evaluated. The linear trend was estimated by number of tooth brushings as a continuous variable ( $0-1$ time per day as 0,2 times as 1 , and $\geq 3$ times as 2 ) and number of missing teeth as a continuous variable ( 0 missing teeth as $0,1-7$ missing teeth as $1,8-14$ missing teeth as 2 , and $\geq 15$ missing teeth as 3 ). Subgroup analyses were performed within demographics and vascular risk factors for the better understanding of oral hygiene effects. Interaction between oral hygiene and each subgroup was tested using a two-sided Wald test in the Cox proportional hazards regression analyses. The statistical analyses were performed using SAS software (version 9.2, SAS Institute, Cary, NC, USA). A $p$ value of less than 0.05 was defined as statistically significant.

To minimise the effect of missing value and selection bias, we performed a further sensitivity analysis using multiple imputation [24]. Missing values were imputed using multivariable imputation by chained equations. This method allows specification of the multivariable structure in the data as a series of conditional imputation models that are adapted to the type of variable. Predictive mean matching was used to impute continuous variables, binary logistic regression was used for dichotomous variables and multinomial logistic regression was used for polytomous variables. Missingness mechanism was assumed as being missing at random [25]. Five imputed datasets were created and the results were combined to account for uncertainty in the imputed data. The multiple imputation was performed using the MICE package in R project, version 3.7.0 (http:/www.r-project.org).

\section{Results}

When demographics were evaluated by standardised differences, female and low socioeconomic status was more frequently noted in excluded subjects (Electronic supplementary material [ESM] Table 1). Among the included subjects, the mean age was 53.3 years, $57.9 \%$ were men, $34.6 \%$ had hypertension, $16.9 \%$ had dyslipidaemia, and $20.5 \%$ were current smokers. Periodontal disease was noted in $17.5 \%$. Based on the self-reported survey, $44.0 \%$ of subjects visited a dental clinic for any reason and $1.1 \%$ were missing 15 or more teeth. Moreover, $42.6 \%$ of the subjects brushed their teeth $\geq 3$ times per day. Approximately $25.9 \%$ of the subjects had visited a dental clinic for professional dental cleaning at least once per year (Table 1).

With a median follow-up of 10.0 years (interquartile range 9.2-11.5), the cohort included 31,545 cases of diabetes. The estimated 10 year event rate was $16.1 \%$ (95\% CI $15.9 \%$, $16.3 \%$ ) for new-onset diabetes. Figure 2 shows the KaplanMeier survival curves for new-onset diabetes according to periodontal disease, number of tooth brushings, dental clinic visits for any reason, professional dental cleaning and number of missing teeth. Presence of periodontal disease $(p<0.001)$ and number of missing teeth $(p<0.001)$ were associated with 
Table 1 Baseline characteristics of the study population

\begin{tabular}{|c|c|}
\hline Characteristics & Total \\
\hline Number of subjects & 188,013 \\
\hline Age (years) & $53.3 \pm 8.6$ \\
\hline Male sex & $108,870(57.9)$ \\
\hline \multicolumn{2}{|l|}{ Socioeconomic status } \\
\hline Fifth quintile (highest) & $75,931(40.4)$ \\
\hline Fourth quintile & $37,478(19.9)$ \\
\hline Third quintile & $26,545(14.1)$ \\
\hline Second quintile & $23,828(12.7)$ \\
\hline First quintile (lowest) & $23,867(12.7)$ \\
\hline Covered by Medical Aid & $364(0.2)$ \\
\hline Alcohol consumption & $82,467(43.9)$ \\
\hline \multicolumn{2}{|l|}{ Smoking status } \\
\hline Never & $130,457(69.4)$ \\
\hline Former smoker & $19,064(10.1)$ \\
\hline Current smoker & $38,492(20.5)$ \\
\hline Regular physical activity & $18,250(9.7)$ \\
\hline \multicolumn{2}{|l|}{ Anthropometric measurements } \\
\hline BMI $\left(\mathrm{kg} / \mathrm{m}^{2}\right)$ & $23.9 \pm 2.8$ \\
\hline Systolic blood pressure $(\mathrm{mmHg})$ & $125.1 \pm 16.4$ \\
\hline Diastolic blood pressure (mmHg) & $78.3 \pm 10.7$ \\
\hline \multicolumn{2}{|l|}{ Comorbidities } \\
\hline Hypertension & $65,063(34.6)$ \\
\hline Dyslipidaemia & 31,707 (16.9) \\
\hline Renal disease & $458(0.2)$ \\
\hline History of malignancy & $22,532(12.0)$ \\
\hline \multicolumn{2}{|l|}{ Medications } \\
\hline Any antihypertensive agents & $51,394(27.3)$ \\
\hline Any lipid-lowering agents & $13,072(7.0)$ \\
\hline \multicolumn{2}{|l|}{ Laboratory findings } \\
\hline Total cholesterol (mmol/l) & $5.12 \pm 0.93$ \\
\hline Fasting blood glucose level (mmol/l) & $5.13 \pm 0.79$ \\
\hline Aspartate aminotransferase ( $\mu \mathrm{kat} / \mathrm{l})$ & $0.44 \pm 0.25$ \\
\hline Alanine aminotransferase $(\mu \mathrm{kat} / \mathrm{l})$ & $0.42 \pm 0.32$ \\
\hline$\gamma$-Glutamyl transferase $(\mu \mathrm{kat} / \mathrm{l})$ & $0.61 \pm 0.83$ \\
\hline Proteinuria ( $\geq 1+$ in dipstick test) & $5314(2.8)$ \\
\hline \multicolumn{2}{|l|}{ Oral health status } \\
\hline Presence of periodontal disease & $32,968(17.5)$ \\
\hline \multicolumn{2}{|l|}{ Number of missing teeth } \\
\hline 0 & $143,203(76.2)$ \\
\hline $1-7$ & $40,157(21.4)$ \\
\hline $8-14$ & $2676(1.4)$ \\
\hline$\geq 15$ & $1977(1.1)$ \\
\hline \multicolumn{2}{|l|}{ Oral hygiene care } \\
\hline Dental visit for any reason & $82,799(44.0)$ \\
\hline \multicolumn{2}{|l|}{ Tooth brushing (times/day) } \\
\hline $0-1$ & $26,013(13.8)$ \\
\hline 2 & $81,921(43.6)$ \\
\hline$\geq 3$ & $80,079(42.6)$ \\
\hline Dental visit for professional cleaning & $48,748(25.9)$ \\
\hline
\end{tabular}

Data are expressed as mean $\pm \mathrm{SD}$ or $n(\%)$ an increased risk of new-onset diabetes, whereas the risk of diabetes occurrence was negatively correlated with professional dental cleaning $(p<0.001)$ and frequent tooth brushing $(p<0.001)$ (Fig. 2).

In the multivariable analysis, presence of periodontal disease was associated with new-onset diabetes in Model 1 (HR 1.05, 95\% CI 1.03, 1.08, $p<0.001$ ), Model 2 (HR 1.08, $95 \%$ CI 1.06, 1.11, $p<0.001)$ and Model 3 (HR 1.09, 95\% CI $1.07,1.12, p<0.001)$. The number of tooth brushings $(\geq 3$ times/day) remained negatively correlated with the occurrence of new-onset diabetes in Model 1 (HR 0.91, 95\% CI 0.88 , $0.94, p<0.001, p$ for trend $<0.001$ ), Model 2 (HR 0.92, $95 \%$ CI $0.89,0.95, p<0.001, p$ for trend $<0.001)$ and Model 3 (HR 0.92, 95\% CI 0.89, 0.95, $p<0.001, p$ for trend $<0.001$ ). Furthermore, number of missing teeth ( $\geq 15$ teeth) also remained positively associated with occurrence of new-onset diabetes in Model 1 (HR 1.19, 95\% CI 1.08, 1.32, $p<0.001, p$ for trend <0.001), Model 2 (HR 1.21, 95\% CI 1.09, 1.33, $p<0.001, p$ for trend $<0.001$ ) and Model 3 (HR 1.21, 95\% CI 1.09, 1.33, $p<0.001, p$ for trend $<0.001$ ) (Table 2).

Professional dental cleaning was not significantly associated with the occurrence of new-onset diabetes in the multivariable analysis, even though this oral hygiene indicator was negatively correlated with the occurrence of new-onset diabetes in the univariable analysis (Table 2).

In the subgroup analysis, the presence of periodontal disease was more strongly associated with new-onset diabetes in the $\leq 51$-years-old group compared with those who were $\geq 52$ years old ( $p$ for interaction $<0.05$ ) in subjects without regular physical activity compared with those with regular physical activity (ESM Table 2). The number of tooth brushings was also more strongly related to new-onset diabetes in the $\geq 52$-years-old group compared with those who were $\leq 51$ years old, in men compared with women, in subjects without regular physical activity compared with those with regular physical activity, and in those with hypertension compared with those without hypertension ( $p$ for interaction $<0.05$ ) (ESM Table 3). The number of missing teeth was more closely associated with new-onset diabetes in the $\geq 52$-yearsold group compared with those who were $\leq 51$ years old ( $p$ for interaction <0.05) (ESM Table 4). There were no statistical interactions regarding the relationship of periodontal disease, number of tooth brushings, and number of teeth lost with newonset diabetes in association with other demographics or comorbidities in the multivariable analysis (ESM Tables 2-4).

The distributions of overall missing data for the baseline dataset and the dataset excluding subjects with a previous diabetes history are noted in ESM Table 5. For the sensitivity analysis with multiple imputation method, 443,264 subjects without a history of diabetes were included. The characteristics of the subjects in the datasets before and after multiple imputation are shown in ESM Table 6. In sensitivity analysis after multiple imputation applying multivariable Model 3, 


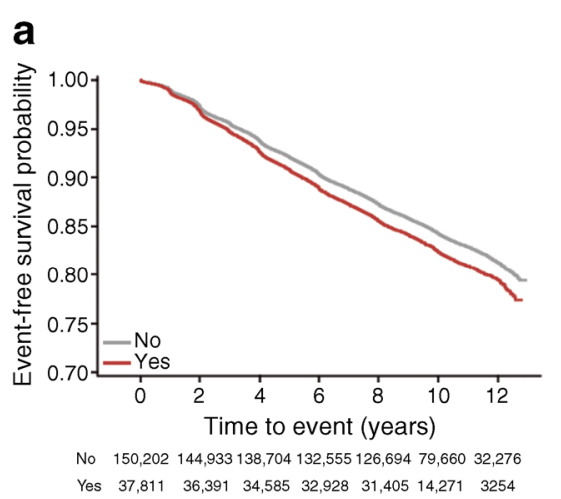

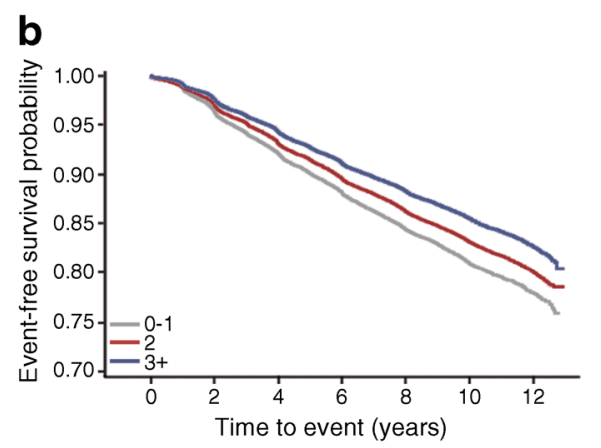

$\begin{array}{llllllll}0-1 & 26,013 & 24,775 & 23,345 & 22,008 & 20,699 & 13,650 & 5547\end{array}$ $\begin{array}{llllllll}2 & 81,921 & 78,788 & 75,147 & 71,658 & 68,304 & 44,087 & 18,433\end{array}$

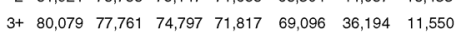

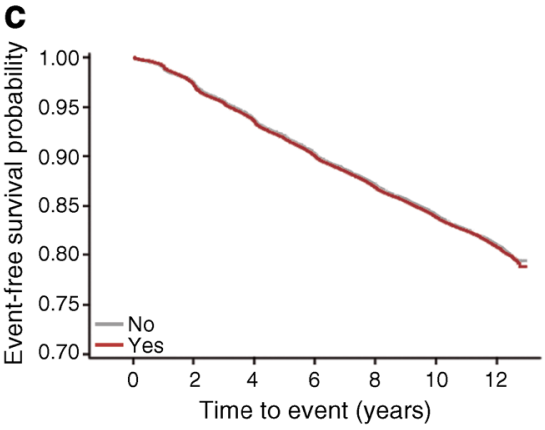

$\begin{array}{llllllll}\text { Yes } 82,799 & 80,013 & 76,543 & 73,178 & 70.035 & 40,234 & 14,501\end{array}$ No $105,214101,31196,746 \quad 92,305 \quad 88,064 \quad 53,697 \quad 21,029$

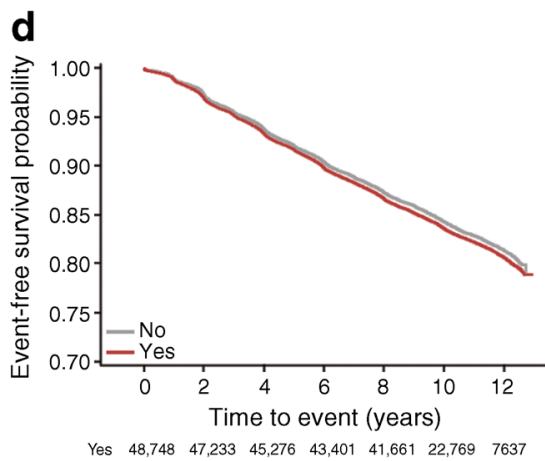

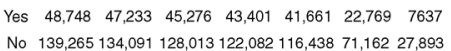

Fig. 2 Kaplan-Meier survival curves associated with oral diseases and oral hygiene behaviours for occurrence of new-onset diabetes. Risk of new-onset diabetes occurrence depended on the presence of periodontal

presence of periodontal disease was associated with newonset diabetes (HR 1.07, 95\% CI $1.051 .09, p<0.001$ ) (ESM Table 7). The number of tooth brushings ( $\geq 3$ times/ day) remained negatively correlated with the occurrence of new-onset diabetes (HR 0.93, 95\% CI 0.89, 0.96, $p<0.001$ ). Furthermore, the number of missing teeth ( $\geq 15$ teeth) showed a marginal association with occurrence of new-onset diabetes (HR 1.20, 95\% CI 0.98, 1.47, $p=0.069$ ] (ESM Table 7).

\section{Discussion}

The key findings of our study are that frequency of tooth brushing was associated with a decreased risk of new-onset diabetes, and the presence of periodontal disease and missing teeth $(\geq 15)$ may augment the risk of new-onset diabetes. In a previous case-control study in Japan, a lower frequency of tooth brushing was associated with a higher odds ratio (OR 1.61) of diabetes [26]. Among youths with type 1 and type 2 diabetes, acceptable diabetes control based on $\mathrm{HbA}_{1 \mathrm{c}}$ was associated with tooth brushing ( $\geq 1$ time daily: OR $3.10,95 \%$ CI 1.26, 7.62) [27]. In terms of periodontal diseases and number of missing teeth, individuals with poorly controlled diabetes $\left(\mathrm{HbA}_{1 \mathrm{c}}>9.0 \%\right)$ have been reported to have severe

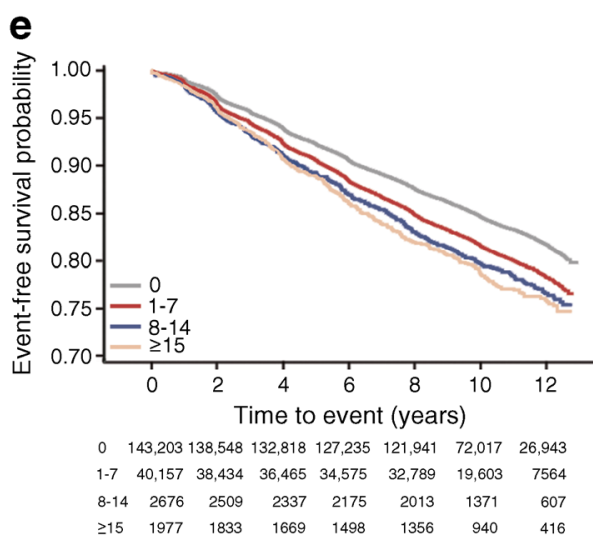

disease (a) $(p<0.001)$, number of tooth brushings (b) $(p<0.001)$, professional dental cleaning (d) $(p<0.001)$, and number of missing teeth $(\mathbf{e})$ $(p<0.001)$, but not dental visits for any reason $(\mathbf{c})(p=0.440)$

periodontal disease more frequently than those without diabetes (OR 2.90, 95\% CI 1.40, 6.03) [28]. Moreover, more severe periodontal disease was associated with the development of glucose intolerance in non-diabetic individuals [29]. A US study reported that adults with diabetes had about twice the number of teeth missing as individuals without diabetes, and one in every five cases of edentulism was related to diabetes [30]. Our results are in concordance with these previous studies and provide additional information on the link between oral hygiene and new-onset diabetes in a longitudinal study in a general population setting.

Nevertheless, in our study the hazard ratios for the development of new-onset diabetes based on the presence of periodontal diseases and number of missing teeth were relatively low compared with those found in previous studies [31]. Similar to our results, in a previous study that defined periodontal disease by ICD-9 code, individuals with new-onset diabetes had a 1.04 times higher risk for periodontal disease compared with individuals without new-onset diabetes [32]. In contrast, in a National/European Health Interview Surveys study, the adjusted odds ratio of periodontal disease for people with diabetes was 1.22 (95\% CI 1.03, 1.45) [31]. Moreover, as discussed above, in a previous US cross-sectional study, people with diabetes reported twice as many missing teeth as 


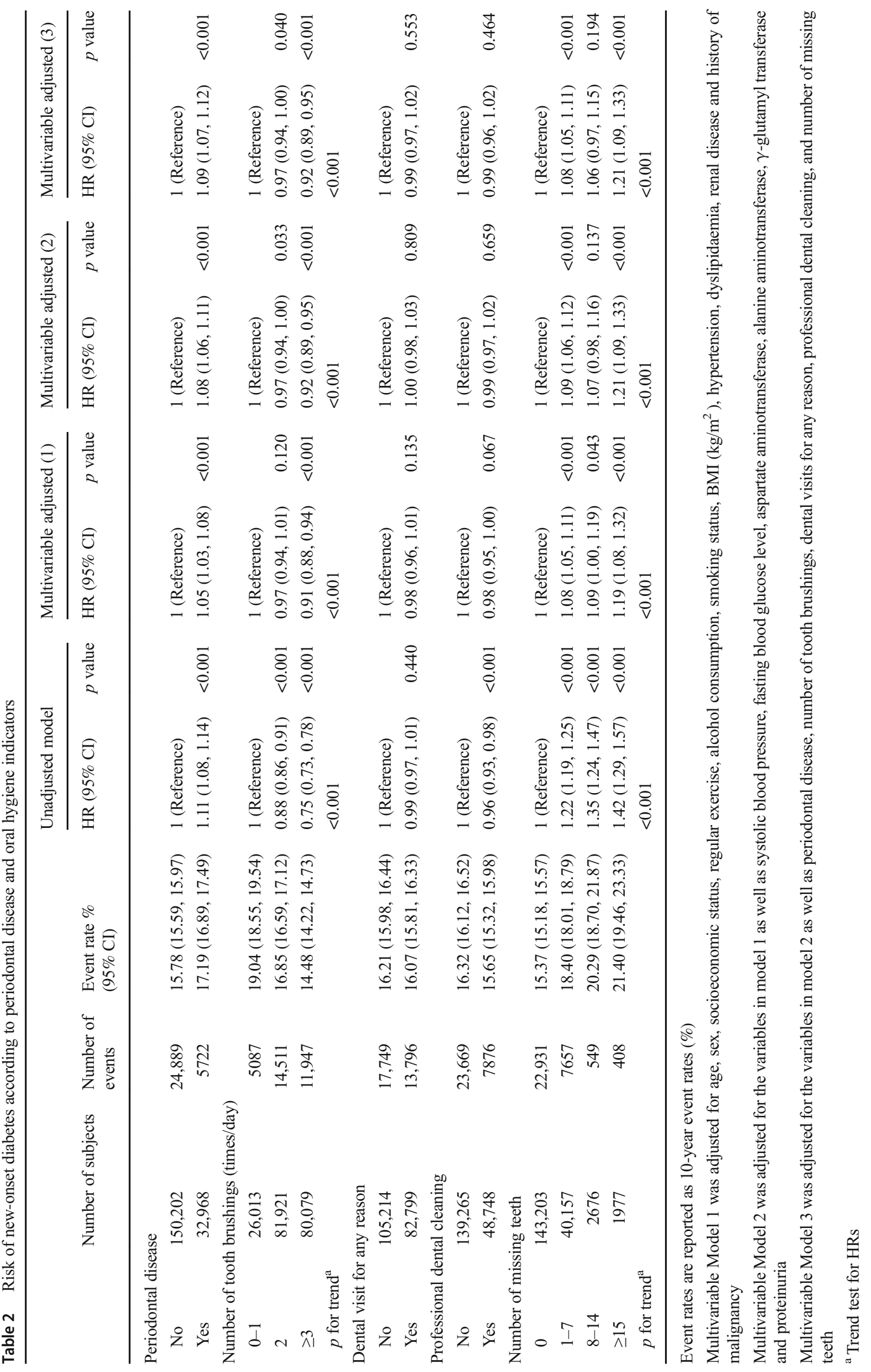


those without diabetes [30]. A recent meta-analysis of epidemiological studies regarding the effect of periodontitis on diabetes showed that there were significant and consistent results supporting the relationship between periodontal disease and worsening of glycaemic control in individuals without diabetes [33]. This discrepancy is likely to be due to differences in study design, population, definition for presence of periodontal disease (for example, whether ICD-10 codes or a validated probing method were used [34]), and methods for defining new-onset diabetes. Furthermore, the subjects included in our study had a higher socioeconomic status than those who were excluded. In a previous study, high socioeconomic status was associated with a low incidence of periodontal disease [35]. The difference in socioeconomic status between the subjects included and excluded in our study may explain the lower HR of the presence of periodontal disease for newonset diabetes.

In our study, professional dental cleaning was negatively associated with the occurrence of new-onset diabetes in the univariable analysis. In a previous case-control study, professional dental cleaning was demonstrated to possibly reduce blood inflammatory biomarker levels and $\mathrm{HbA}_{1 \mathrm{c}}$ [36]. The effectiveness of professional dental cleaning for glycaemic control was also demonstrated in individuals with type 2 diabetes in a small randomised controlled trial [37]. Even though our results are in line with these previous studies, the associations of dental visits for any reason and professional dental cleaning with the occurrence of new-onset diabetes were no longer statistically significant in our dataset after adjusting for various important confounding factors. These results suggest that other confounding factors are more strongly related than professional dental cleaning to the occurrence of new-onset diabetes.

In our subgroup analysis, the strength of the association of periodontal disease, number of tooth brushings and number of missing teeth with new-onset diabetes varied according to age (above and below 52 years old, the median age). Although it is difficult to give a clear reason for this relationship, we assume that genetic factors, stress, and many other general diseases may have different effects on periodontal disease, oral hygiene indicators and new-onset diabetes depending on age.

Although our study did not identify the precise mechanism underlying the relationship between number of missing teeth and new-onset diabetes, the following findings could explain this association. Poor oral hygiene may be related to the chronic inflammatory process, which affects the supporting structures of the teeth. Ulceration in periodontal pockets and teeth loss provide easy access for the translocation of oral bacteria into the systemic circulation [38], and dysbiosis of the oral biofilm with high virulence leads to indirect induction of proinflammatory cytokines [39]. The cytokines increase the inflammatory burden both locally and systemically. Moreover, inflammatory biomarkers are elevated in advanced stages of poor oral hygiene because of systemic inflammation [40]. Previous studies have reported that an increased level of inflammatory biomarkers is independently associated with insulin insensitivity and new-onset type 2 diabetes [41, 42]. Accordingly, the potentiated inflammatory reaction from poor oral hygiene may evoke impaired glycaemic control. Moreover, endotoxin lipopolysaccharide (LPS), a component of oral bacterial cell walls, induces an innate immune reaction. A previous study showed that blood LPS levels and LPS activity were significantly increased in individuals with type 2 diabetes compared with those without type 2 diabetes [43].

Our current study has several limitations. First, our results cannot be generalised to other ethnicities because our dataset only includes subjects from the Korean population. Second, because information on oral hygiene indicators was acquired from a self-reported questionnaire, there may be recall bias. Third, the definition of the presence of periodontal disease based on the ICD-10 code using health claim data does not reflect recently published case definitions and classification criteria for periodontal disease [34]. Fourth, in our dataset, different degrees of severity of periodontal disease could not be investigated. Fifth, although our study was performed with health claim data regarding dental health check-ups, the information of detailed probing depths, attachment loss, and bleeding after probing was lacking. Sixth, because our study design was retrospective and observational, serial changes in dental health information during the study period from 2003 to 2006 were not investigated. The study design could also cause a selection bias, and therefore direct causal relationships cannot be conclusively determined. Seventh, we could not exclude all subjects with underlying diabetes because the $\mathrm{HbA}_{1 \mathrm{c}}$ and oral glucose tolerance tests were not performed during health check-ups. Eighth, because our dataset came from health claim data, it was difficult to clearly distinguish between type 1 and type 2 diabetes. Ninth, we could not identify the exact cause of missing teeth through health examination records. Tenth, educational level, marital status and blood inflammatory markers, such as C-reactive protein, were not evaluated because NHIS-HEALS did not include this information. Finally, the exclusion of many subjects may have also led to selection bias.

In conclusion, frequent tooth brushing may be an attenuating factor for the risk of new-onset diabetes, and the presence of periodontal disease and increased number of missing teeth may be augmenting factors. Improving oral hygiene may be associated with a decreased risk of occurrence of new-onset diabetes.

Data availability The data that support the findings of this study are available from NHIS-HEALS, but restrictions apply to the availability of these data, which were used under licence for the current study and so are not publicly available. Data are, however, available from the authors upon reasonable request and with permission from the National Health Insurance System. 
Funding This project was supported by a grant from the Basic Science Research Program through the National Research Foundation of Korea funded by the Ministry of Education (2018R1D1A1B07040959 to T-JS). The funders had no role in study design, data collection and analysis, the decision to publish, or preparation of the manuscript.

Authors' relationships and activities The authors declare that there are no relationships or activities that might bias, or be perceived to bias, their work.

Contribution statement $\mathrm{YC}, \mathrm{KJL}, \mathrm{HGW}$, and TJS, contributed to conception and design of the study, data acquisition, analysis and interpretation of results, and drafted and critically revised the manuscript; JSL, contributed to design of the study, data acquisition and interpretation, and critically revised the manuscript. TJS accepts full responsibility for the work and/or the conduct of the study, had access to the data, and controlled the decision to publish. All authors gave final approval for the manuscript to be published.

\section{References}

1. Ma RC, Chan JC (2013) Type 2 diabetes in East Asians: similarities and differences with populations in Europe and the United States. Ann N Y Acad Sci 1281:64-91. https://doi.org/10.1111/nyas.12098

2. Rhee EJ (2015) Diabetes in Asians. Endocrinol Metab (Seoul) 30: 263-269

3. Beckman JA, Creager MA, Libby P (2002) Diabetes and atherosclerosis: epidemiology, pathophysiology, and management. JAMA 287(19):2570-2581. https://doi.org/10.1001/jama.287.19. 2570

4. Fox CS, Golden SH, Anderson C et al (2015) Update on prevention of cardiovascular disease in adults with type 2 diabetes mellitus in light of recent evidence: a scientific statement from the American Heart Association and the American Diabetes Association. Diabetes Care 38(9):1777-1803. https://doi.org/10.2337/dci150012

5. Kassebaum NJ, Smith AGC, Bernabe E et al (2017) Global, region$\mathrm{al}$, and national prevalence, incidence, and disability-adjusted life years for oral conditions for 195 countries, 1990-2015: a systematic analysis for the global burden of diseases, injuries, and risk factors. J Dent Res 96(4):380-387. https://doi.org/10.1177/ 0022034517693566

6. Kinane DF, Stathopoulou PG, Papapanou PN (2017) Periodontal diseases. Nat Rev Dis Primers 3:17038

7. Lissowska J, Pilarska A, Pilarski P et al (2003) Smoking, alcohol, diet, dentition and sexual practices in the epidemiology of oral cancer in Poland. Eur J Cancer Prev 12(1):25-33. https://doi.org/ 10.1097/00008469-200302000-00005

8. Chang JS, Lo HI, Wong TY et al (2013) Investigating the association between oral hygiene and head and neck cancer. Oral Oncol 49: $1010-1017$

9. Guha N, Boffetta P, Wunsch Filho V et al (2007) Oral health and risk of squamous cell carcinoma of the head and neck and esophagus: results of two multicentric case-control studies. Am J Epidemiol 166:1159-1173

10. Park SY, Kim SH, Kang SH et al (2019) Improved oral hygiene care attenuates the cardiovascular risk of oral health disease: a population-based study from Korea. Eur Heart J 40(14):11381145. https://doi.org/10.1093/eurheartj/ehy836

11. Martin-Cabezas R, Seelam N, Petit C et al (2016) Association between periodontitis and arterial hypertension: a systematic review and meta-analysis. Am Heart J 180:98-112
12. Mealey BL, Rose LF (2008) Diabetes mellitus and inflammatory periodontal diseases. Curr Opin Endocrinol Diabetes Obes 15:135141

13. Pitsavos C, Tampourlou M, Panagiotakos DB et al (2007) Association Between low-grade systemic inflammation and type 2 diabetes mellitus among men and women from the ATTICA Study. Rev Diabet Stud 4:98-104

14. Chapple IL, Genco R, Working group 2 of joint EFPAAPw (2013) Diabetes and periodontal diseases: consensus report of the Joint EFP/AAP Workshop on Periodontitis and Systemic Diseases. J Clin Periodontol 40(Suppl 14):S106-S112

15. Preshaw PM, Alba AL, Herrera D et al (2012) Periodontitis and diabetes: a two-way relationship. Diabetologia 55(1):21-31. https:// doi.org/10.1007/s00125-011-2342-y

16. Forner L, Larsen T, Kilian M, Holmstrup P (2006) Incidence of bacteremia after chewing, tooth brushing and scaling in individuals with periodontal inflammation. J Clin Periodontol 33(6):401-407. https://doi.org/10.1111/j.1600-051X.2006.00924.x

17. Song SO, Jung CH, Song YD et al (2014) Background and data configuration process of a nationwide population-based study using the Korean national health insurance system. Diabetes Metab J 38: 395-403

18. Seong SC, Kim YY, Park SK et al (2017) Cohort profile: the National Health Insurance Service-National Health Screening Cohort (NHIS-HEALS) in Korea. BMJ Open 7:e016640

19. Kim MK, Han K, Joung HN, Baek KH, Song KH, Kwon HS (2019) Cholesterol levels and development of cardiovascular disease in Koreans with type 2 diabetes mellitus and without preexisting cardiovascular disease. Cardiovasc Diabetol 18:139

20. Lee KH, Lee CM, Kwon HT, Oh S-W (2010) Relationship between obesity and smoking in Korean men: data analyses from the third and fourth Korea National Health and Nutrition Examination Surveys (KNHANES). J Korean Soc Res Nicotine Tobacco 1: $115-123$

21. Lee JH, Choi JK, Jeong SN, Choi SH (2018) Charlson comorbidity index as a predictor of periodontal disease in elderly participants. J Periodontal Implant Sci 48(2):92-102. https://doi.org/10.5051/jpis. 2018.48.2.92

22. Lee JH, Lee JS, Park JY et al (2015) Association of lifestyle-related comorbidities with periodontitis: a nationwide cohort study in Korea. Medicine (Baltimore) 94:e1567

23. Chang Y, Woo HG, Park J, Lee JS, Song TJ (2019) Improved oral hygiene care is associated with decreased risk of occurrence for atrial fibrillation and heart failure: a nationwide population-based cohort study. Eur J Prev Cardiol:2047487319886018

24. van Buuren S, Groothuis-Oudshoorn K (2011) MICE: Multivariate Imputation by Chained Equations in R. J Stat Softw 45:1-67

25. Rubin DB (1976) Inference and missing data. Biometrika. 63:581592

26. Fujita M, Ueno K, Hata A (2009) Lower frequency of daily teeth brushing is related to high prevalence of cardiovascular risk factors. Exp Biol Med (Maywood) 234:387-394

27. Merchant AT, Oranbandid S, Jethwani M et al (2012) Oral care practices and A1c among youth with type 1 and type 2 diabetes. $\mathrm{J}$ Periodontol 83:856-863

28. Tsai C, Hayes C, Taylor GW (2002) Glycemic control of type 2 diabetes and severe periodontal disease in the US adult population. Community Dent Oral Epidemiol 30(3):182-192. https://doi.org/ 10.1034/j.1600-0528.2002.300304.x

29. Saito T, Shimazaki Y, Kiyohara Y et al (2004) The severity of periodontal disease is associated with the development of glucose intolerance in non-diabetics: the Hisayama study. J Dent Res 83(6): 485-490. https://doi.org/10.1177/154405910408300610

30. Luo H, Pan W, Sloan F, Feinglos M, Wu B (2015) Forty-year trends in tooth loss among American adults with and without diabetes mellitus: an age-period-cohort analysis. Prev Chronic Dis 12:E211 
31. de Miguel-Infante A, Martinez-Huedo MA, Mora-Zamorano E et al (2018) Periodontal disease in adults with diabetes, prevalence and risk factors. Results of an observational study. Int J Clin Pract: e13294

32. Lee CY, Kuan YH, Tsai YF, Tai CJ, Tsai TH, Huang KH (2019) Correlation between diabetes mellitus and periodontitis in Taiwan: a nationwide cohort study. Diabetes Res Clin Pract 150:245-252

33. Graziani F, Gennai S, Solini A, Petrini M (2018) A systematic review and meta-analysis of epidemiologic observational evidence on the effect of periodontitis on diabetes: an update of the EFP-AAP review. J Clin Periodontol 45(2):167-187. https://doi.org/10.1111/ jcpe. 12837

34. Caton JG, Armitage G, Berglundh T et al (2018) A new classification scheme for periodontal and peri-implant diseases and conditions - Introduction and key changes from the 1999 classification. J Periodontol 89(Suppl 1):S1-S8

35. Thomson WM, Sheiham A, Spencer AJ (2000) Sociobehavioral aspects of periodontal disease. Periodontol 2012(60):54-63

36. Soorya KV, Suchetha A, Lakshmi P et al (2014) The effect of scaling and root planing on glycaemic control, periodontal status and gingival crevicular fluid TNF-alpha levels in an Indian population- to reveal the ambivalent link. J Clin Diagn Res 8: ZC22-ZC26

37. Zhang H, Li C, Shang S, Luo Z (2013) Scaling and root planing with enhanced root planing on healthcare for type 2 diabetes mellitus: a randomized controlled clinical trial. J Dent Sci 8:272280
38. Hajishengallis G (2015) Periodontitis: from microbial immune subversion to systemic inflammation. Nat Rev Immunol 15(1): 30-44. https://doi.org/10.1038/nri3785

39. Loos BG (2005) Systemic markers of inflammation in periodontitis. J Periodontol 76(11 Suppl):2106-2115. https://doi.org/10.1902/ jop.2005.76.11-S.2106

40. Noack B, Genco RJ, Trevisan M, Grossi S, Zambon JJ, De Nardin E (2001) Periodontal infections contribute to elevated systemic Creactive protein level. J Periodontol 72(9):1221-1227. https://doi. org/10.1902/jop.2000.72.9.1221

41. Festa A, D’Agostino R Jr, Howard G, Mykkanen L, Tracy RP, Haffner SM (2000) Chronic subclinical inflammation as part of the insulin resistance syndrome: the Insulin Resistance Atherosclerosis Study (IRAS). Circulation 102(1):42-47. https:// doi.org/10.1161/01.cir.102.1.42

42. Pradhan AD, Manson JE, Rifai N, Buring JE, Ridker PM (2001) Creactive protein, interleukin 6 , and risk of developing type 2 diabetes mellitus. JAMA 286(3):327-334. https://doi.org/10.1001/jama. 286.3.327

43. Jayashree B, Bibin YS, Prabhu D et al (2014) Increased circulatory levels of lipopolysaccharide (LPS) and zonulin signify novel biomarkers of proinflammation in patients with type 2 diabetes. Mol Cell Biochem 388:203-210

Publisher's note Springer Nature remains neutral with regard to jurisdictional claims in published maps and institutional affiliations. 